\title{
X-ray Magnetic Circular Dichroism of Valence Fluctuating State in Eu at High Magnetic Fields
}

\author{
Y. H. Matsuda,, , Z Z. W. Ouyang, ${ }^{1}$ H. Nojiri, ${ }^{1}$ T. Inami, ${ }^{2}$ \\ K. Ohwada, ${ }^{2}$ M. Suzuki,${ }^{3}$ N. Kawamura, ${ }^{3}$ A. Mitsuda, ${ }^{4}$ and H. Wada ${ }^{4}$ \\ ${ }^{1}$ Institute for Materials Research, Tohoku University, \\ 2-1-1 Katahira, Aoba-ku, Sendai 980-8577, Japan \\ 2 Synchrotron Radiation Research Unit, Japan Atomic Energy Agency, Sayo, Hyogo 679-5148, Japan \\ ${ }^{3}$ SPring-8/JASRI,1-1-1 Kouto, Sayo, Hyogo 679-5198 Japan \\ 4 Department of Physics, Kyushu University, Fukuoka 812-8581, Japan
}

(Dated: October 29, 2018)

\begin{abstract}
X-ray magnetic circular dichroism (XMCD) at the Eu $L$-edge $(2 p \rightarrow 5 d)$ in two compounds exhibiting valence fluctuation, namely $\mathrm{EuNi}_{2}\left(\mathrm{Si}_{0.18} \mathrm{Ge}_{0.82}\right)_{2}$ and $\mathrm{EuNi}_{2} \mathrm{P}_{2}$, has been investigated at pulsed high magnetic fields of up to $40 \mathrm{~T}$. A distinct XMCD peak corresponding to the trivalent state $\left(\mathrm{Eu}^{3+} ; f^{6}\right)$, whose ground state is nonmagnetic $(J=0)$, was observed in addition to the main XMCD peak corresponding to the magnetic $(J=7 / 2)$ divalent state $\left(\mathrm{Eu}^{2+} ; f^{7}\right)$. This result indicates that the $5 d$ electrons belonging to both valence states are magnetically polarized. It was also found that the ratio $P_{5 d}(3+) / P_{5 d}(2+)$ between the polarization of $5 d$ electrons $\left(P_{5 d}\right)$ in the $\mathrm{Eu}^{3+}$ state and that of $\mathrm{Eu}^{2+}$ is $\sim 0.1$ in $\mathrm{EuNi}_{2}\left(\mathrm{Si}_{0.18} \mathrm{Ge}_{0.82}\right)_{2}$ and $\sim 0.3$ in $\mathrm{EuNi}_{2} \mathrm{P}_{2}$ at magnetic fields where their macroscopic magnetization values are the same. The possible origin of the XMCD of the $\mathrm{Eu}^{3+}$ state and an explanation of the dependence of $P_{5 d}(3+) / P_{5 d}(2+)$ on the material are discussed in terms of hybridization between the conduction electrons and the $f$ electrons.
\end{abstract}

PACS numbers: 71.20.Eh, 71.27.+a, 75.30.Mb, 78.70.Dm

At low temperatures, where thermal excitations are suppressed, quantum effects become clear and intriguing phenomena such as quantum phase transitions are observed in high magnetic fields [1, 2]. However, microscopic measurement techniques are limited in the presence of high magnetic fields. For example, photoemission experiments at high magnetic fields are very difficult to perform. A synchrotron X-ray is an element- and shell-selective microscopic probe, and X-ray magnetospectroscopy is a powerful method for studying electronic states at high magnetic fields. Recently, X-ray absorption spectroscopy (XAS) at high magnetic fields up to $40 \mathrm{~T}$ has been carried out in order to clarify fieldinduced valence transitions of $\mathrm{Yb}$ - and $\mathrm{Eu}$-based intermetallic compounds by using a pulsed magnet [3, 4]. As for the research of magnetic materials, X-ray magnetic circular dichroism (XMCD) spectroscopy is an even more powerful technique than XAS. Since high-magnetic-field XMCD can be used for examining antiferromagnetic and paramagnetic materials [5] as well as ferromagnetic materials, various kinds of field-induced phenomena, such as metamagnetic transitions, can be studied microscopically.

Valence fluctuation phenomena found in rare-earth intermetallic compounds have been attracting considerable attention from the viewpoint of the strong correlation of electrons. In the valence fluctuation state at low temperatures, the Kondo effects becomes significant and the ground state is nonmagnetic in many cases. When magnetic fields are applied, the localized magnetic moment tends to appear. In fact, field-induced valence transitions are found along with the metamagnetism in some systems exhibiting valence fluctuation, such as $\mathrm{Ce}_{0.8} \mathrm{La}_{0.1} \mathrm{Th}_{0.1}$ [6], $\mathrm{YbInCu}_{4}$ [7], $\mathrm{EuNi}_{2}\left(\mathrm{Si}_{1-x} \mathrm{Ge}_{x}\right)_{2}$ [8]. In order to elucidate the valence fluctuation phenomena, the XMCD experiment is highly intriguing. We can examine the magnetic polarization of electrons in different valence states determined by the magnetic fields.

In this Letter, we present the application of high-magnetic-field XMCD to $\mathrm{EuNi}_{2}\left(\mathrm{Si}_{0.18} \mathrm{Ge}_{0.82}\right)_{2}$ and $\mathrm{EuNi}_{2} \mathrm{P}_{2}$, which are typical materials exhibiting valence fluctuation [9, 10]. The magnetic polarization of the $\mathrm{Eu}$ $5 d$ electrons in different valence states is examined by using XMCD spectroscopy at high magnetic fields. The dependence of the polarization of the $5 d$ electrons on the field and the material is discussed in terms of hybridization between the conduction and $f$ electrons $(c-f$ hybridization).

The XMCD experiment with Eu $L_{2,3}$-edge $\left(2 p_{1 / 2,3 / 2} \rightarrow 5 d\right)$ was carried out at high magnetic fields at BL39XU in SPring-8 11] by using a miniature pulsed magnet producing fields of up to $40 \mathrm{~T}[3,4]$. A polycrystal of $\mathrm{EuNi}_{2}\left(\mathrm{Si}_{0.18} \mathrm{Ge}_{0.82}\right)_{2}$ and a single crystal of $\mathrm{EuNi}_{2} \mathrm{P}_{2}$ were used. The crystals were powdered and diluted in order to achieve an effective sample thickness of about $10 \mu \mathrm{m}$ for the transmission measurement. An experimental setup for high-field XMCD spectroscopy was similar to that for high-field XAS, described in Ref. [3, 4]. A diamond X-ray phase plate was introduced into the present setup to generate circularly polarized X-rays. A storage oscilloscope was used to record the detector output voltages corresponding to the incident 


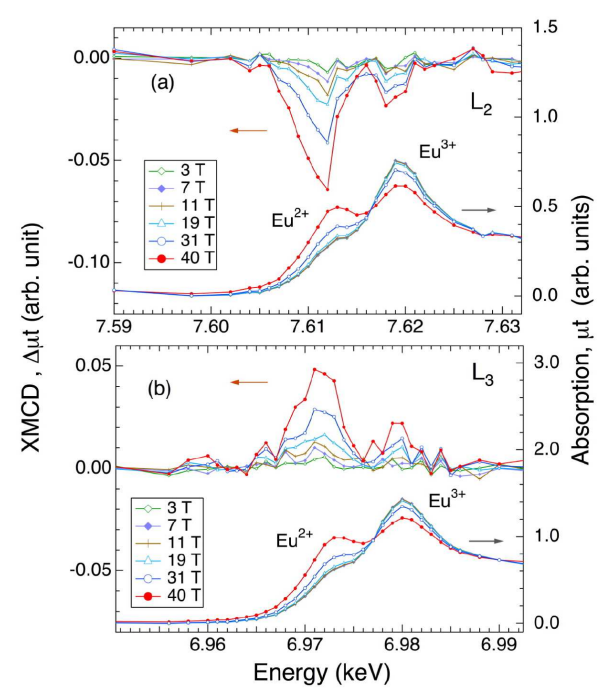

FIG. 1: (a) XMCD and XAS spectra at various magnetic fields at $5 \mathrm{~K}$ for the $L_{2}$ edge. XAS spectra are plotted in such a way that the absorption at $7.598 \mathrm{keV}$ becomes zero. (b) $\mathrm{XMCD}$ and XAS spectra at various magnetic fields at $5 \mathrm{~K}$ for the $L_{3}$ edge. XAS spectra are plotted in such a way that the absorption at $6.956 \mathrm{keV}$ becomes zero.

and transmitted X-ray intensities of the sample as a function of time, together with the pulsed magnetic field. The XMCD signal $\left(\Delta \mu t=\mu^{+} t-\mu^{-} t\right)$ is determined as the difference in the absorption intensities for right$\left(\mu^{+} t\right)$ and left- $\left(\mu^{-} t\right)$ circular polarization. The $\mu^{+} t$ and $\mu^{-} t$ were measured by successive two shots of a pulsed field at a fixed X-ray energy, as the photon helicity was reversed every shot.

The measured XMCD spectra of $\mathrm{EuNi}_{2}\left(\mathrm{Si}_{0.18} \mathrm{Ge}_{0.82}\right)_{2}$ are shown in Fig. 1 together with the XAS spectra at several magnetic fields. The XAS spectra of the $L_{2}$ and $L_{3}$ absorption edges are very similar, except for the intensities. The valence fluctuation manifests itself as a doublepeak structure in the XAS spectra [12]. The higher energy XAS peak, which is shown in Fig. 1, is attributed to the $\mathrm{Eu}^{3+}$ state, while the lower energy peak is attributed to the $\mathrm{Eu}^{2+}$ state $[8,9]$. The XAS spectrum strongly depends on the magnetic field, exhibiting a valence change induced by the magnetic field [4, 13]. We found that the double-peak structure corresponding to the two valence states is observed in the XMCD spectra as well as in the XAS spectra. The double peaks indicate that the electrons in the $\mathrm{Eu} 5 d$ orbital, where the excited electron finally goes in the $L_{2,3}$ transitions, are magnetically polarized not only in the $\mathrm{Eu}^{2+}$ state $\left(f^{7} ; J=7 / 2\right.$ in the ground state), but also in the $\mathrm{Eu}^{3+}$ state $\left(f^{6} ; J=0\right.$ in the ground state).

Figure 2 shows the dependence of the integrated intensity of the XMCD peak $\left(I_{m c d}\right)$ on the magnetic field, as well as the magnetic polarization of the $5 d$ electrons $\left(P_{5 d}\right)$. The degree of magnetic polarization of the $\mathrm{Eu}$

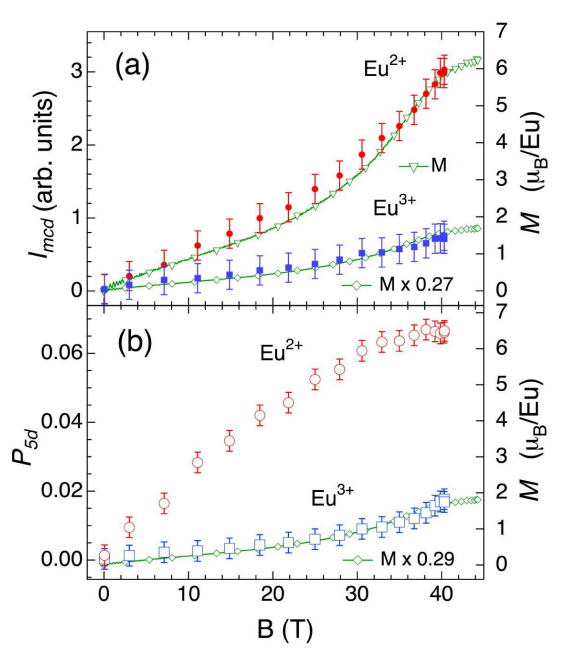

FIG. 2: (a) Magnetic field dependence of the integrated XMCD intensities of $\mathrm{Eu}^{2+}$ and $\mathrm{Eu}^{3+}$ states for the $L_{2}$ edge. (b) The degree of magnetic polarization of $5 d$ electrons is plotted as a function of the magnetic field. Magnetization measured at $4.2 \mathrm{~K}[14]$ is shown for comparison.

$5 d$ electrons in each valence state is defined in this paper as $P_{5 d}=\int \Delta \mu t d E / \int \mu t d E$, where $\Delta \mu t$ and $\mu t=$ $\left(\mu^{+} t+\mu^{-} t\right) / 2$ are the XMCD intensity and the absorption intensity, respectively. $\int \Delta \mu t d E\left(=I_{m c d}\right)$ is deduced by integration of the XMCD peak shown in Fig. 1 for each valence state. $\int \mu t d E$ is obtained from the integrated intensity of the absorption peak. Curve fitting analysis is performed in order to evaluate the absorption intensities $\int \mu t d E=I_{2}$ and $I_{3}$ for the $\mathrm{Eu}^{2+}$ and $\mathrm{Eu}^{3+}$ states, respectively, at different magnetic fields. The Eu valence $v^{*}$ is directly deduced from $v^{*}=2 I_{2} /\left(I_{2}+I_{3}\right)+3 I_{3} /\left(I_{2}+I_{3}\right)$ [12]. The details of the curve fitting are shown in Ref. 4 . The $v^{*}$ values at $5 \mathrm{~K}$ obtained in the present study namely 2.74 at $0 \mathrm{~T}$ and 2.47 at $40 \mathrm{~T}$ are in good agreement with the field dependence of $v^{*}$ reported in our previous paper [4]. The magnetization $(M)$ of the sample at $4.2 \mathrm{~K}[14$ is shown in Fig. 2 in order to compare it with the dependence of $I_{m c d}$ and $P_{5 d}$ on the field. An increase of the magnetization caused by the valence change is observed at around $35 \mathrm{~T}[8,14]$. In addition, $I_{m c d}$ of $\mathrm{Eu}^{2+}$ and that of $\mathrm{Eu}^{3+}$ scale together with the magnetization, as expected for XMCD experiments. However, $P_{5 d}$ is qualitatively different for the $\mathrm{Eu}^{2+}$ and $\mathrm{Eu}^{3+}$ states. If we define the $P_{5 d}$ of $\mathrm{Eu}^{2+}\left(f^{7}\right)$ and that of $\mathrm{Eu}^{3+}\left(f^{6}\right)$ as $P_{5 d}(2+)$ and $P_{5 d}(3+)$, respectively, it is found that the dependence of $P_{5 d}(2+)$ on the field is convex upward and exhibits saturation more clearly than the magnetization at around $40 \mathrm{~T}$, while $P_{5 d}(3+)$ still appears to follow the magnetization curve. Since the magnetic field dependence of $I_{m c d}$ and $P_{5 d}$ for the $L_{3}$-edge is found to be qualitatively similar to that for the $L_{2}$-edge, we only show and discuss the results of the $L_{2}$-edge in this Letter.

In Fig. 3(a), we show the results for another sam- 

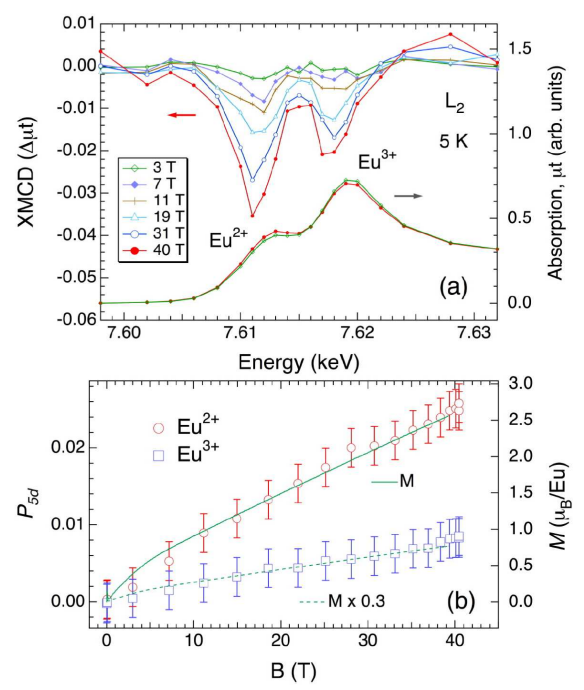

FIG. 3: (a)XMCD spectra in $\mathrm{EuNi}_{2} \mathrm{P}_{2}$ at high magnetic fields. The XAS spectra at 3 and $40 \mathrm{~T}$, which are plotted in such a way that the absorption intensity becomes zero at $7.598 \mathrm{eV}$, are also shown. (b) The degree of magnetic polarization of the $5 d$ electrons, as well as the magnetization measured at $4.2 \mathrm{~K}$, are plotted as functions of the magnetic field.

ple, namely $\mathrm{EuNi}_{2} \mathrm{P}_{2}$. The $\mathrm{Eu}$ valence in $\mathrm{EuNi}_{2} \mathrm{P}_{2}$ is $2.5-2.6[10,15]$, which is almost exactly midway between 2 and 3 , due to the strong hybridization, and this material is considered to be characterized by stronger hybridization than $\mathrm{EuNi}_{2}\left(\mathrm{Si}_{0.18} \mathrm{Ge}_{0.82}\right)_{2}$. We found two clear peaks in the XAS and XMCD spectra, as in the case of $\mathrm{EuNi}_{2}\left(\mathrm{Si}_{0.18} \mathrm{Ge}_{0.82}\right)_{2}$. Only small changes in the XAS spectra are visible even at $40 \mathrm{~T}$, and the Eu valence depends only slightly on the magnetic field in the case of $\mathrm{EuNi}_{2} \mathrm{P}_{2}(2.64$ at $0 \mathrm{~T}$ and 2.61 at $40 \mathrm{~T})$. The deduced polarization of the $5 d$ electrons $\left(P_{5 d}\right)$ for each valence state is plotted as a function of the field in Fig. 3 (b) together with the magnetization. It is found that both $P_{5 d}$ values follow the magnetization.

In the following paragraphs, we discuss the XMCD spectra and the deduced polarization $P_{5 d}$. The double-peak structure found in the XMCD spectra of $\mathrm{EuNi}_{2}\left(\mathrm{Si}_{0.18} \mathrm{Ge}_{0.82}\right)_{2}$ and $\mathrm{EuNi}_{2} \mathrm{P}_{2}$ indicates that the $\mathrm{Eu}$ $5 d$ electrons are magnetically polarized not only in the $\mathrm{Eu}^{2+}$ state, but also in the $\mathrm{Eu}^{3+}$ state. Since the ground state of $\mathrm{Eu}^{2+}\left(f^{7}\right)$ is magnetic $(J=7 / 2 ; L=0, S=7 / 2)$, we expect significant XMCD for the $\mathrm{Eu}^{2+}$ state. However, the ground state of $\mathrm{Eu}^{3+}\left(f^{6}\right)$ is nonmagnetic $(J=0$; $L=3, S=3)[8,9]$, and it is not obvious why there is a finite XMCD signal for the $\mathrm{Eu}^{3+}$ state. Possible origins for the XMCD signal of the $\mathrm{Eu}^{3+}$ state include (i) hybridization of the $\mathrm{Eu}^{2+}$ and $\mathrm{Eu}^{3+}$ states in the final state, (ii) effect of the excited state of $\mathrm{Eu}^{3+}$ state $(J=1)$, and (iii) magnetic polarization of the conduction electrons.

Regarding the first option (i), we can reasonably assume that there is hardly any mixing of the valence in the final state of the $L_{2,3}$ transitions. This is due to the fact that the energy difference between the $5 d$ electrons in $\mathrm{Eu}^{3+}$ and $\mathrm{Eu}^{2+}$ is around $8 \mathrm{eV}$ owing to the strong Coulomb interaction between the core-hole and the $f^{n}$ electrons ( $n=6$ or 7$)$. Therefore, it can be assumed that each absorption peak can be attributed to a nearly pure $\mathrm{Eu}^{3+}$ or $\mathrm{Eu}^{2+}$ state. In this case, the possibility of obtaining XMCD due to the $\mathrm{Eu}^{2+}$ component from the absorption assigned to the $\mathrm{Eu}^{3+}$ state is almost zero.

Since the first excited state of $\mathrm{Eu}^{3+}$ is magnetic $(J=1)$ [8, 9], the second possible origin should have some effects. The energy separation between the first excited and ground states is about $480 \mathrm{~K}[8]$. When we estimate the magnetization due to the Van Vleck paramagetism using $1.43 \times 10^{-6}\left(\mu_{B} / \mathrm{Eu}^{3+}\right) / \mathrm{T}$ as the magnetic susceptibility [16], the magnetization of $\mathrm{Eu}^{3+}$ is estimated to be about $0.6 \mu_{B} / \mathrm{Eu}^{3+}$ at $40 \mathrm{~T}$. This value is in good agreement with the Van Vleck term reported in a previous work on $\mathrm{Eu}_{3} \mathrm{Fe}_{5} \mathrm{O}_{12}$ [17]. If we take the Van Vleck term of $\mathrm{Eu}^{3+}$ into account, the relative contribution of the magnetization between the two valence states at fields lower than $20 \mathrm{~T}$ (below the transition field of $\left.\mathrm{EuNi}_{2}\left(\mathrm{Si}_{0.18} \mathrm{Ge}_{0.82}\right)_{2}\right), M(3+) / M(2+)$ is assumed to be around 0.09 for $\mathrm{EuNi}_{2} \mathrm{P}_{2}$ and 0.06 for $\mathrm{EuNi}_{2}\left(\mathrm{Si}_{0.18} \mathrm{Ge}_{0.82}\right)_{2}$. It appears that these values are two or three times smaller than the ratio $P_{5 d}(3+) / P_{5 d}(2+)$, as we show below.

However, in compounds exhibiting valence fluctuation, the mixing between the $\mathrm{Eu}^{3+}: J=0$ and $\mathrm{Eu}^{3+}: J=1$ states can be enhanced by the strong $c-f$ hybridization. According to a previous work on $\mathrm{EuCu}_{2} \mathrm{Si}_{2}[18]$, significant contribution of the $\mathrm{Eu}^{3+}: J=1$ state to the magnetic properties was observed. Therefore, the effect of $\mathrm{Eu}^{3+}: J=1$ state is one of the plausible origins of the $\mathrm{Eu}^{3+}-\mathrm{XMCD}$ observed in the present work.

The third possible origin is that the conduction electrons are polarized by strong hybridization with the $4 f$ electrons in the magnetic $\mathrm{Eu}^{2+}$ states, and these polarized conduction electrons induce the magnetic polarization of the $5 d$ electrons in the nonmagnetic $\mathrm{Eu}^{3+}$ state. Owing to the strong $c$ - $f$ hybridization, this mechanism also can be regarded as plausible. Moreover, this postulated mechanism is similar to that found in $\mathrm{DyLu}$, where magnetic polarization of the $5 d$ electrons in nonmagnetic $\mathrm{Lu}\left(f^{14}\right)$ in DyLu was observed by magnetic resonance scattering [19]. Here, it should be stressed that the strength of the $c-f$ hybridization plays an important role for the XMCD of the $\mathrm{Eu}^{3+}$ state not only in the second possible origin but also in the third possible origin.

Next, we compare the relative magnitude $P_{5 d}(3+) / P_{5 d}(2+)$ of the polarization of $5 d$ electrons between $\mathrm{EuNi}_{2} \mathrm{P}_{2}$ and $\mathrm{EuNi}_{2}\left(\mathrm{Si}_{0.18} \mathrm{Ge}_{0.82}\right)_{2}$ in the same magnetization range, $1<M /\left(\mu_{B} / \mathrm{Eu}\right)<3$. From Figs. 2(b) and 3(b), we obtain $P_{5 d}(3+) / P_{5 d}(2+)=0.30 \pm 0.03$ and $0.12 \pm 0.03$ for $\mathrm{EuNi}_{2} \mathrm{P}_{2}$ and $\mathrm{EuNi}_{2}\left(\mathrm{Si}_{0.18} \mathrm{Ge}_{0.82}\right)_{2}$, respectively. Those values are nearly constant in the 
interval $1<M /\left(\mu_{B} / \mathrm{Eu}\right)<3$. The ratio of the values for the two materials is $0.30 / 0.12=2.5$. This dependence on the material suggests that the induced polarization of the $5 d$ electrons in the $\mathrm{Eu}^{3+}$ state depends on the electronic structure, as well as possibly on the strength of the $c-f$ hybridization.

If we assume a simple two-level scheme for describing the hybridization and denote the energy gap between the two valence states as $\Delta E$, the hybridization energy parameter $V$ can be evaluated from the Eu valence $v^{*}$ at low temperatures and zero magnetic fields. If we use $v^{*}=2.6$ for $\mathrm{EuNi}_{2} \mathrm{P}_{2}$ and 2.8 for $\mathrm{EuNi}_{2}\left(\mathrm{Si}_{0.18} \mathrm{Ge}_{0.82}\right)_{2}$, it is found that $V / \Delta E=2.3$ and 0.7 for $\mathrm{EuNi}_{2} \mathrm{P}_{2}$ and $\mathrm{EuNi}_{2}\left(\mathrm{Si}_{0.18} \mathrm{Ge}_{0.82}\right)_{2}$, respectively. The relative value of the hybridization strength between the two materials is thus estimated to be $2.3 / 0.7$, which is around 3.3. Therefore, we found that the relative value of $P_{5 d}(3+) / P_{5 d}(2+)$ in the two materials (2.5) is close to the estimated relative hybridization value in the two materials (3.3). This agreement might support our assumption that $\mathrm{Eu}^{3+}$ $\mathrm{XMCD}$ is induced through the $c-f$ hybridization.

Finally, we discuss the dependence of $P_{5 d}$ in $\mathrm{EuNi}_{2}\left(\mathrm{Si}_{0.18} \mathrm{Ge}_{0.82}\right)_{2}$ on the magnetic field. Since the field dependence of $P_{5 d}(3+)$ seems to follows the macroscopic magnetization curve, as seen in Fig. 2, it appears that $P_{5 d}(3+)$ reflects the magnetization contributed from many Eu sites through the $c$ - $f$ hybridization. Hence, it is likely that the magnetic moments due to the $J=7 / 2$ $\left(\mathrm{Eu}^{2+}\right)$ and $J=1\left(\mathrm{Eu}^{3+}\right)$ states of neighboring $\mathrm{Eu}$ sites induce the polarization of $5 d$ electrons of $\mathrm{Eu}^{3+}$ state. Regarding the $\mathrm{Eu}^{2+}$ state, since the $5 d$ electrons are magnetically polarized by the local $d-f$ exchange interaction, $P_{5 d}(2+)$ directly reflects the magnetic polarization of the $4 f$ electrons in the $\mathrm{Eu}^{2+}$ state. Hence, we regard the dependence of $P_{5 d}(2+)$ on the magnetic field as a hypothetical magnetization curve when all Eu sites have localized $J=7 / 2$ magnetic moments. It is noteworthy that the magnetization curve of antiferromagnetic $\mathrm{EuNi}_{2}\left(\mathrm{Si}_{0.05} \mathrm{Ge}_{0.95}\right)_{2}$ [8] is similar to that of the dependence of $P_{5 d}(2+)$ on the magnetic field.

In conclusion, valence-selective XMCD has been clearly observed in Eu-based compounds exhibiting valence fluctuation, namely $\mathrm{EuNi}_{2}\left(\mathrm{Si}_{0.18} \mathrm{Ge}_{0.82}\right)_{2}$ and $\mathrm{EuNi}_{2} \mathrm{P}_{2}$, for fields of up to $40 \mathrm{~T}$. Finite XMCD is observed for both valence states $\mathrm{Eu}^{2+}$ and $\mathrm{Eu}^{3+}$, although the expected magnetic properties are very different, i.e., the ground state of $\mathrm{Eu}^{2+}$ has $J=7 / 2$, while that of $\mathrm{Eu}^{3+}$ has $J=0$. We have proposed possible explanations for the XMCD of the nonmagnetic $\mathrm{Eu}^{3+}$ state in terms of hybridization between the conduction electrons and the $4 f$ electrons ( $c$ - $f$ hybridization) ; the two possible origins are (1) mixing of $\mathrm{Eu}^{3+} J=1$ state through the $c-f$ hybridization and (2) spin polarization of the conduction electrons due to the hybridization effect. Another intriguing finding concerns the fact that the dependence of the magnetic polarization of the $5 d$ electrons $\left(P_{5 d}\right)$ on the magnetic field is qualitatively different for the $\mathrm{Eu}^{2+}$ and $\mathrm{Eu}^{3+}$ states at magnetic fields higher than the valence transition field of $\mathrm{EuNi}_{2}\left(\mathrm{Si}_{0.18} \mathrm{Ge}_{0.82}\right)$. This difference is attributed to the different origins of the XMCD, where the $\mathrm{Eu}^{2+} \mathrm{XMCD}$ is caused by localized electrons, while $\mathrm{Eu}^{3+} \mathrm{XMCD}$ reflects the character of the itinerant electrons. Although the detailed mechanism of the XMCD in Eu-based materials exhibiting valence fluctuation is still unclear, it is plausible that the $c$ - $f$ hybridization plays an important role. In addition, actually, recent photoemission experiments reported evidence suggesting strong hybridization between $\mathrm{Eu} 4 f$ and $\mathrm{Ni} 3 d$ electrons in $\mathrm{EuNi}_{2} \mathrm{P}_{2}[20]$. Hence, the conduction electrons referred to in the present XMCD paper can be Ni $3 d$ electrons. For the better understanding of the phenomenon, a theoretical model beyond a single atomic picture 21] might be required.

This work is partly supported by a Grant-in-Aid for Scientific Research on Priority Area "High Field Spin Science in 100 T" (No.451) provided by the Ministry of Education, Culture, Sports, Science and Technology (MEXT), Japan. Y.H.M. and H.N. thank Prof. I. Harada, Prof. A. Kotani and Prof. T. Ziman for fruitful discussions.

* Also at PRESTO, Japan Science and Technology Agency, Saitama 332-0012; Present address, Institute for Solid State Physics, University of Tokyo, Chiba 277-8581; Electronic address: ymatsuda@issp.u-tokyo.ac.jp

[1] N. Harrison et al., Phy. Rev. Lett. 90, 096402 (2003).

[2] A. J. Millis et al., Phy. Rev. Lett. 88, 217204 (2002).

[3] Y. H. Matsuda et al., J. Phys. Soc. Jpn. 76, 034702 (2007).

[4] Y. H. Matsuda et al., J. Phys. Soc. Jpn. 77, 054713 (2008).

[5] O. Mathon et al., J. Synchrotron Rad. 14, 409 (2007).

[6] F. Drymiotis et al., J. Phys.:Condens. Matter 17, L77 (2005).

[7] K. Yoshimura et al., Phy. Rev. Lett. 60, 851 (1988).

[8] H. Wada et al., J. Phys.: Condens. Matter 9, 7913 (1997).

[9] G. Wortmann et al., Phys. Rev. B 43, 5261 (1991).

[10] R. Nagarajan et al., J. Magn. Magn. Mater. 47\&48, 413 (1985).

[11] H. Maruyama et al., J. Synchrotron Rad. 6, 1133 (1999).

[12] G. Wortmann, Hyperfine Interact. 47, 179 (1989).

[13] A. Scherzberg et al., Solid State Commun. 49, 1027 (1984).

[14] A. Mitsuda, PhD Thesis (Kyoto University, 1999).

[15] B. Perscheid et al., J. Magn. Magn. Mater. 47\&48, 410 (1985).

[16] A. Frank, Phy. Rev. 39, 119 (1932).

[17] M. Mizumaki et al., Phy. Rev. B 71, 134416 (2005).

[18] J. Röhler et al., Phys. Rev. Lett. 49, 65 (1982).

[19] B. A. Everitt et al., Phys. Rev. Lett. 75, 3182 (1995).

[20] S. Danzenbacher et al., Phys. Rev. Lett. 102, 026403 (2009).

[21] A. Kotani, Phys. Rev. B 78, 195115 (2008). 